Wojciech KALINOWSKI*

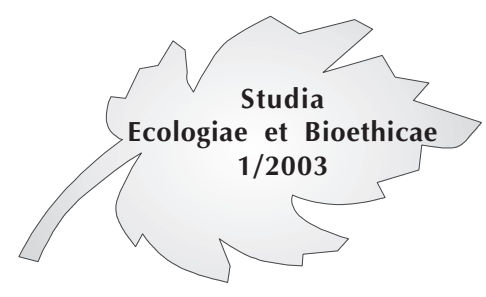

\title{
Eine Grundlage zur Kommunikativen Theologie
}

\section{Was ist Kommunikation?}

Der Begriff Kommunikation (von lat. ",communicatio", Mitteilung) bezeichnet in der Regel zwischenmenschliche Mitteilung und Verständigung mittels bedeutungshaltiger verbaler und non-verbaler Zeichen. Als „dialogisches Wesen" ist der Mensch, um leben, ja sogar um psychisch wie physisch überleben zu können, auf Kommunikation angewiesen. Mittels Kommunikation kann er in Gemeinschaft Mensch werden und sein. Kommunikation erschließt in Form alltäglicher Interaktionen den Kommunikationspartnerinnen eine gemeinsame Welt, in der sie als aktive Subjekte miteinander kommunizieren, Normen und Werte entwickeln und so ihre Identität gewinnen. Kommunikation hat also eine wirklichkeitsstiftende und -erhaltende Macht: „Die subjektive Wirklichkeit von etwas, das nie besprochen wird, fängt allmählich an, hinfällig zu werden"1 ${ }^{\prime 1}$ Eine in dieser Form beschriebene Bedrohung des Menschen durch Wirklichkeitsverlust gründet auf einer konstruktivistischen Position. Sie bringt uns insofern ein Stück weiter, als sie deutlich macht, dass die Wirklichkeit, in der Menschen leben, durch Kommunikation zustande kommt und dass Menschen unbedingt darauf angewiesen sind, mit anderen Menschen deren je andere Wirklichkeiten auszutauschen bzw. sich mit ihnen zu verständigen. Konsequenz einer solchen konstruktivistischen Auffassung könnte aber auch sein, dass sich der Mensch eine völlig illusionäre Wirklichkeit zurechtrichten kann, aus der er, wenn sie von anderen geteilt wird, nicht mehr heraus kommt. Totalitäre Systeme, wie sie sich in geschlossenen Gruppen äußern können, sind Beispiele für eine derartige "Wirklichkeits" erzeugung.

Konstruiert sich nicht gerade auch der glaubende Mensch eine Welt, die zwar seiner Sehnsucht entspricht, die es aber in Wirklichkeit nicht gibt? Eine Glaubenskommunikation, die den Himmel offen hält, die sich also nicht in zwischenmenschlicher Kommunikation und wechselseitiger

\footnotetext{
Wydział Teologiczny Uniwersytetu Warmińsko-Mazurskiego w Olsztynie i Wyższe Seminarium Duchowne w Ełku.

1 Berger P. L., Luckmann T., Die gesellschaftliche Konstruktion der Wirklichkeit. Eine Theorie der Wissenssoziologie, Frankfurt a. M. 1982, 164.
} 
Vergewisserung erschöpft, sondern die Gottesbeziehung in das Kommunikationsgeschehen und -Verständnis mit einschließt, steht unter einem besonderen Illusionsverdacht. Man kann die Herausforderung des Konstruktivismus auch so formulieren:

Kann sich der Mensch dem radikalen Relativismus des Konstruktivismus mit seinen unendlich vielen Wirklichkeiten möglicherweise erst dann stellen, wenn er aus einer unbedingten Gewissheit heraus kommuniziert, dass sein ganzes Leben und auch das der Anderen letztlich von einem ganz Anderen gehalten und getragen ist; und das nicht nur im Moment, sondern als Ganzes und selbst durch den Tod hindurch? Eine solche Gewissheit ist nicht nur eine rationale Einsicht, sie ist eine Beziehungsgewissheit, die - wie wir aus vielen Beispielen biblischer Beterinnen erkennen können - Zweifel, Angst, Trauer, Kampf, Wut, Verlassenheit, Einsamkeit in die Beziehung mit einschließt.

Damit wird deutlich: Kommunikation, wie wir sie verstehen, beschränkt sich keineswegs auf den Informationsaustausch. Sie erschöpft sich nicht im verbalen Ausdruck. Was Menschen tief berührt, was sie unbedingt betrifft, kommunizieren sie in der Regel symbolisch-rituell: in Riten und Gesten, in Bildern und Erzählungen oder über symboltiefe .Gegenstände'. Auch das gemeinsame Tun, das Teilen von Freude und Sorge, von Lust und Verantwortung hat eine tiefe kommunikative Qualität.

Wie wir bereits kurz erwähnt haben, kann uns auf philosophisch-soziologischer Ebene J. Habermas Theorie des "kommunikativen Handelns" im spätmodernen Sprachgewirr um Kommunikation zunächst ein Stück weiterhelfen: In aller Kürze soll seine Unterscheidung von drei Formen des menschlichen Kommunikationshandelns in Erinnerung gerufen werden:

- Das instrumentelle, erfolgsorientierte nicht-soziale Handeln.

- Das strategische, erfolgsorientierte soziale Handeln.

- Das kommunikative, verständigungsorientierte soziale Handeln. Nur das kommunikative Handeln gibt dem Menschen Raum zum Subjektsein und unterstellt ihn nicht dem Nutzen und dem Kalkül.

Der philosophisch-soziologische Begriff des kommunikativen Handelns wurde verschiedentlich theologisch interpretiert. Als solcher ist er offen für die Deutung der dialogischen Grundstruktur des Menschen als Ausdruck seiner Geschöpflichkeit, Gottebenbildlichkeit und Freiheit: Demgemäß ist der Mensch ein immer schon - letztlich von Gott - angesprochenes Wesen, das in Freiheit antworten, aber sich auch verweigern kann. Das Kommunikationsverständnis radikalisiert sich angesichts einer Kommunikationsgemeinschaft, welche in christlicher Hoffnung auch die Toten umfasst, für deren Subjektsein die Lebenden nichts mehr tun können, für die aber die rettende Wirklichkeit Gottes eintritt. ${ }^{2}$

2 Vgl. Peukert H., Wissenschaftstheorie - Handlungstheorie - Fundamentale Theologie. Analysen zu Ansatz, und Status theologischer Theoriebildung, Dusseldorf 1976, 311. 


\section{Epochale Veränderungen in der Kommunikation}

Wie aus den bisherigen Überlegungen bereits deutlich wurde, stehen als Kommunikationsmedien längst nicht mehr nur das gesprochene Wort oder die "Sprache des Körpers” zur Verfügung. Die Kommunikationsmedien erweitern sich ständig. Die Kommunikationsmöglichkeiten und das Kommunikationsausmaß sind in einer epochalen Veränderung begriffen. Wir gehen einer "placeless society”, einer „ortlosen Gesellschaft” entgegen, prophezeit der britische Zukunftsforscher W. Knoke in seinem Buch „Kühne neue Welt”3. Raum und Zeit werden keine Rolle mehr spielen; die Vernetzung der Informationen dürfte zum Nabel der Welt werden. In der "kühnen neuen Welt” des 21. Jahrhunderts werden sich Nationalstaaten, Unternehmen, Gewerkschaften in ihrer heutigen Form ebenso auflösen wie Schulen, Religionen und Familien. Entfernungen werden unbedeutsam; mit einem Mausklick kann jederzeit jede beliebige Information unkontrolliert abgerufen oder weitergegeben, jede Ware bestellt, das elektronische Geld verwaltet werden. Großkonzerne, welche gegenwärtig noch die Wirtschaft bestimmen, werden zerfallen; quer über den Globus schließen sich in „Amöbenform” kleine Unternehmen zusammen und bieten die Leistungen der Großen von heute an. Ein Großteil der Arbeit wird aus den Betrieben ausgelagert und vom privaten Netzanschluss aus zu erledigen sein, auch die Bildung. Wie einst die Menschen zur Schule gingen, so wird in Zukunft die Information zu den Menschen kommen. „Dass die Figur des Lehrers zu einer fast mythischen Größe heranwachsen, dass selbst die geschmähte Betriebsgemeinschaft ein Sehnsuchtsziel werden, dass sich die Studenten nach dem persönlichen Unterrichtsgespräch drängen könnten, von dem sie in den Erzählungen ihrer Eltern gehört haben, ist nur allzu wahrscheinlich" ${ }^{4}$.

$\mathrm{Zu}$ den großen Verlierern der neuen Entwicklung gehören alle jene, welche nicht zeitgerecht in die globale Kommunikation einsteigen können, das sind bei uns die medialen Analphabeten. Wenn schon jetzt die Armut weiblich ist, dann wird sich auch die diesbezügliche Teilung der Gesellschaft weiterhin verschärfen: „Nur zehn Prozent aller NetzNutzer sind, Schätzungen zufolge, weiblich", schreibt S. Gaschke. Sie hält den „vernetzten Computer" für „ein überschätztes Männerspielzeug”5. Die größten Verlierer werden die zahllosen nicht vernetzbaren Arbeit-

3 KNoKe W., Kühne neue Welt. Leben in der „placeless society“ des 21. Jahrhunderts, deutsch: Wien 1996

4 Frühwald W., Vor uns die Cyber-Sinflut, in: Der Mensch in Netz. Kommerz und Chaos in der digitalen Welt, ZEIT Punkte 5/96, 12.

5 GaschKe S., Frauen und Technik, in: Der Mensch in Netz. Kommerz und Chaos in der digitalen Welt, ZEIT Punkte 5/96, 17. 
skräfte in den Ländern der sogenannten dritten und vierten Welt sein, deren Potential völlig unbrauchbar wird.

Die Entwicklung in Richtung einer globalen, virtuellen Kommunikationsgesellschaft wird eine Identitätsveränderung der Menschen bewirken. Wer an die Kommunikationsmedien angeschlossen ist, wird eine schier unendliche Freiheit haben, mit jenen Menschen zu kommunizieren, welche die eigenen Interessen teilen; dies freilich nicht in unmittelbaren persönlichen Begegnungen, sondern als wechselseitiger Austausch von Informationen in einer illusionären Gemeinschaft. Der Kommunikationstheoretiker N. Bolz (Essen) diagnostizierte bei den „39. Salzburger Gesprächen" als eine der Paradoxien der neuen Medienwelt: Aufgrund der menschlichen Lust nach Kommunikativität steigern die technischen Medien die Kommunikationsaktivitäten des Menschen, ohne sich dabei etwas zu sagen. Zwischenmenschliche, "face-to-face"-Kommunikation wird zukünftig nur mehr im Kontext dieser neuen Kommunikationsmöglichkeiten denkbar sein.

\section{Der Mensch ,kann nicht nicht kommunizieren”}

In diesem ersten „metakommunikativen Axiom” fasst der bekannte Kommunikationsforscher P. Watzlawick ${ }^{6}$ pointiert zusammen, womit sich die sozialwissenschaftliche Kommunikationsforschung seit den dreißiger Jahren des 20. Jahrhunderts beschäftigt: nämlich mit der menschlichen Kommunikation als einer sozialen Grundfähigkeit zur interaktiven Vermittlung bedeutungsvoller Inhalte durch den Austausch kulturell geprägter Zeichen. Die aus der Propagandaforschung hervorgegangenen sozialwissenschaftlichen Kommunikationstheorien richten ihr Interesse immer stärker auf den inzwischen zunehmend technisierten Kommunikationsprozess via E-Mail, Internet, Worid Wide Web usw. Davon sind die Theorien der intrapersönlichen und der zwischenmenschlichen Kommunikation zu unterscheiden.

\subsection{Sender und Empfänger}

Eine Grundannahme in den sozialwissenschaftlichen Kommunikationstheorien besteht darin, dass ein "Sender" eine von ihm kodierte Botschaft mittels eines Mediums mit einem Empfänger austauscht, der den Inhalt der Botschaft dekodiert, um ihn zu verstehen ${ }^{7}$. Dieses Sender-

6 Watzlawick P., (Beavin J. H.,) Jackson Don D., Menschliche Kommunikation: Formen, Störungen, Paradoxien, Bern 1969, 53.

7 Vgl. Schulz von Hun, Miteinander reden. Störungen und Klarungen. Allgemeine Psychologie der Kommunikation, Reibek, 1981. 
Empfängermodell kann in begrenzter Weise aufklären, dass Inhalte niemals neutral vermittelt werden können. Sowohl der Information Sendende als auch der Empfangende beziehen sich auf Grund ihrer Entwicklungs- und Sozialisationserfahrungen auf ein Bedeutungsspektrum, das in vieler Hinsicht vieldeutig sein kann. So kommt es, dass eine Botschaft völlig anders ankommen kann, als sie vom Sender gemeint war. Speziell die Paartherapie und Konzepte von Kommunikationstrainings haben sich diese Erkenntnisse zu Nutze gemacht, um Kommunikationsbarrieren aufzuklären und überwinden zu helfen. Wie wir vor allem im Zusammenhang mit der Darstellung der Kommunikation nach dem Ansatz der Themenzentrierten Interaktion zeigen werden, greift das Sender-EmpfängerModell bei weitem zu kurz, um die Dynamik lebendiger Kommunikation erklären zu können. Wir werden sehen, dass alle Ebenen der Kommunikation, die Sache, die kommuniziert wird, die Subjekte, ihre Interaktion und Kommunikation wie auch der konkrete Kontext in „dynamischer Balance" aufeinander bezogen sind. Das Sender-Empfänger-Modell wird der Vielschichtigkeit von Kommunikationsakten nicht gerecht.

\subsection{Kommunizieren von Anfang an}

Watzlawicks Axiom, dass der Mensch, „wie immer er es versuchen mag", "nicht nicht kommunizieren" 8 kann, entspricht unserer Erfahrung von Beginn des Lebens an. Tatsächlich ist jeder Mensch ein von Anfang an auf Kommunikation hin angelegtes und von gelingender menschlicher Kommunikation zutiefst abhängiges Wesen; dies auch dann noch, wenn sie/er in der Abgeschiedenheit eines kontemplativen Ordens leben und die verbale Kommunikation mit Menschen auf ein Minimum einschränken würde. Gerade ein solch religiöser Kontext erweitert den Kommunikationsbegriff. Denn sowohl die nonverbale Kommunikation der Menschen untereinander wie auch die Kommunikation im und mit dem geheimnisvoll verborgenen, aber dem Menschen dennoch unendlich nahen $\mathrm{Du}$, die wir Beten nennen, wird vermutlich auch das Personsein dieses Menschen bestimmen. Menschliche Kommunikation ist für Menschen so entscheidend, dass frühe Kommunikationsstörungen oder -Verweigerungen zu schweren seelischen und körperlichen Schädigungen, ja zum Tod von Menschen führen können ${ }^{9}$.

Entwicklungs- und Sozialpsychologien haben für die pränatalen und frühkindlichen Lebensphasen lange Zeit eine weitgehend einseitige Kommunikation der Mutter und anderer früher Bezugspersonen mit dem

Watzlawick, Menschliche Kommunikation, 51.

9 Vgl. u.a. Spitz R. A., Vom Säugling zum Kleinkind. Naturgeschichte der Mutter-Kind-Beziehung im ersten Lebensjahr, 1996. 
Fötus bzw. mit dem Säugling oder Kleinkind angenommen. Demgegenüber nehmen neuere Forschungen bereits für die frühesten Lebensphasen eine wechselseitige Kommunikation ${ }^{10}$ an. Die Mutter und andere frühe Bezugspersonen beeinflussen nicht nur das Kind, es gilt auch umgekehrt: Schon das Kind im Mutterleib kommuniziert mit der Mutter und über sie mit der Umwelt, sodass es auch auf diese Umwelt aktiven Einfluss ausüben kann. Welche schwangere Frau weiß nicht, wie sehr ihr Kind das Leben bestimmen kann? Kinder sind von der liebenden Zuwendung ihrer frühen Bezugspersonen abhängig. Es gilt aber auch, dass sie in der Kommunikation zwischen den Generationen, welche mit der Kommunikation zwischen Mutter und Kind in der Schwangerschaft beginnt und das ganze Leben fortdauert, nicht einfach nur die geliebten, verwöhnten, vernachlässigten, missbrauchten Objekte Erwachsener sind. In der neueren Pädagogik wird auch schon dem sehr jungen Kind, ja dem Embryo, Subjekthaftigkeit und Intersubjektivität zuerkannt. Für zwischenmenschliche Kommunikation gilt, wenn sie menschenwürdig sein soll, dass immer Menschen Menschen begegnen. Menschliche Kommunikation ist eine "Ich-Du" - bzw. eine "Ich-Wir" - Beziehung.

\subsection{Der Mensch, ein dialogisches Wesen}

Die Beziehungsgestalt menschlicher Kommunikation hat der jüdische Philosoph Martin Buber mit der Beschreibung des Menschen als dialogisches Wesen erfasst. M. Buber unterscheidet, wenn es um Begegnung geht, die "Grundworte" „Ich-Du" und „Ich-Es":

Wer Du spricht, hat kein Etwas zum Gegenstand. Denn wo Etwas ist, ist anderes Etwas, jedes Es grenzt an andere Es, Es ist nur dadurch, dass es an andere grenzt. Wo aber Du gesprochen wird, ist kein Etwas. Du grenzt nicht. Wer Du spricht, hat kein Etwas, hat nichts. Aber er steht in der Beziehung ${ }^{11}$.

Kein Mensch darf also in der menschlichen Kommunikation zum „Etwas”, zum Objekt des anderen gemacht werden, wenn Begegnung gelingen soll. „Pädagogisch fruchtbar ist nicht die pädagogische Absicht, sondern die pädagogische Begegnung", sagt Buber. Diese dialogische Anthropologie wird in der Themenzentrierten Interaktion nach R. C. Cohn positiv aufgegriffen und um die Gruppen-, die Sach-und die Kontextebene erweitert. Zur besonderen Herausforderung wird im Hinblick auf das "Es", auf die Sache; also auf den Gegenstand der Glaubenskommunikation und der Theologie. Geht es hier auch um eine, Ich -

10 Vgl. u.a. Beniamin J., Die Fesseln der Liebe. Psychoanalyse, Feminismus und das Problem der Macht. Frankfurt a.M. 1993.

11 Buber M., Ich und Du, Heidelberg 1997, $10 \mathrm{f}$. 
$D u$, oder doch um eine Ich - Es Beziehung? Vermutlich kann beides der Fall sein: Das Kommunizieren von Glaubensgehalten bzw. theologischen Inhalten wird einer Ich - Es Beziehung entsprechen. Wenn sich der Mensch aber dem unendlichen Geheimnis Gottes stellt und in die betende oder feiernde Kommunikation eintritt, die ja - etwa nach dem ostkirchlichen Verständnis - Theologie erst ermöglicht, dann wird diese Begegnung in Analogie zur menschlichen "Ich-Du"-Beziehung gedacht werden dürfen. Die bekannte chassidische Erzählung von der Bekehrung des Knaben gibt eine Ahnung von solch tiefer Kommunikation wieder.

Rabbi Ahron kam einst in die Stadt, in der der kleine Mordechai, der nachmalige Rabbi von Lechowitz, aufwuchs. Dessen Vater brachte ihm den Knaben und klagte, dass er im Lernen keine Ausdauer habe. „Lasst ihn mir eine Weile hier", sagte Rabbi Ahron. Als er mit dem kleinen Mordechai allein war, legte er sich hin und bettete das Kind an sein Herz. Schweigend hielt er es am Herzen, bis der Vater kam. "Ich habe ihm ins Gewissen geredet", sagte er, "hinfort wird es ihm an Ausdauer nicht fehlen."

Wenn der Rabbi von Lechowitz diese Begebenheit erzählte, fügte er hinzu: „Damals habe ich gelernt, wie man Menschen bekehrt”"12.

\subsection{Gott - ein kommunikatives Wesen}

Die bisherigen Überlegungen drängen uns dazu, über Gott als das Subjekt, d. h. als den Ausgangs-, bleibenden Bezugs- und Zielpunkt jeden theologischen Bemühens nachzudenken. Es ist schon deutlich geworden, dass die entscheidenden (konstitutiven) und kommunikativen Handlungen der Glaubensgemeinschaft Gott selbst zum Subjekt haben, jedenfalls in einem primären und einem fundamentalen Sinn. Außerdem ist deutlich geworden, dass selbst eine ganz theozentrische Theologie wie die dialektische Theologie Barths nicht umhin kommt, auch vom Menschen zu reden, wenn sie von Gott redet. Dies heißt wiederum, umgekehrt geblickt, von Gott als einen auf den Mensch ausgerichteten Gott zu sprechen.

Es mag zunächst ein wenig befremden und Nachfragen provozieren, wenn wir deshalb an dieser Stelle von Gott als einem kommunikativen Wesen sprechen. Der Verdacht könnte sein, dass hier Gott wieder für ein theologisches Konzept vereinnahmt würde. Spiegelung menschlicher Kommunikationserfahrungen und -Sehnsüchte in Gott hinein verfallen. Diese Gefahr ist tatsächlich immer gegeben, und kann nur durch die Durchführung des Konzepts selber widerlegt werden. So weit sollten Missverständnisse allerdings schon ausgeschlossen sein, die von einem

$\overline{12}$ Buber M., Die Erzahlungen der Chassidim, Zürich 1949, 327. 
eingeschränkten Begriff der Kommunikation ausgehen und deshalb an dieser Stelle argwöhnen könnten, uns ginge es um die Sanktionierung des bloßen Redens über Gott. Stille vor Gott, Meditation, Gottesdienst in verschiedener Art, Glaubensgespräch, Bibel teilen waren und sind feste Bestandteile unserer Kurse Kommunikativer Theologie. Das heißt, es geht uns gerade um ein integratives Verständnis von Kommunikation, welches das Reden über Gott mit dem Reden von Gott und mit dem Reden $\mathrm{zu}$ Gott verbindet.

Eine weitere ernst zu nehmende Anfrage an das Konzept der Kommu-nikativen Theologie könnte in diesem Zusammenhang lauten: Wird nicht das Anderssein, das Fremdsein Gottes übersehen? Erste Teilantwort: Ja, Gott ist auch einer, der schweigt, einer, der so redet, dass Menschen ihn nicht verstehen, einer, der sich gerade dem Menschen, welcher sich vertrauensvoll an ihn wendet, entziehen kann. Gott ist also kein Gott, der am Ende einer Telefonleitung zu jeder Zeit erreichbar wäre! Eine Theologie, welche nicht berücksichtigt, dass Gott immer auch der ganz Andere, der Schweigende, der Verborgene und sich Entziehende ist, verfehlt ihre Aufgabe. Zweite Teilantwort: Gerade dies bedeutet aber nicht, dass Theologie selber ganz fremd, ganz anders, ganz verborgen sei oder sich in Schweigen hüllen soll. Theologie hat nun einmal die Aufgabe, auch da zu reden, wo es darauf aufmerksam zu machen gilt, dass hier jede Rede verstummen muss. Ansonsten könnte leicht die Gefahr bestehen, dass das Denken und Reden vorzeitig eingestellt wird. Es ist gewiss richtig, dass es auf ganz entscheidende Fragen der Theologie, etwa auch auf die Frage, wie Gott und das Leid zu vereinbaren sind, keine unsere menschliche Vernunft befriedigende Antwort gibt. Aufgabe der Theologie ist aber nicht, deshalb über diese Frage zu schweigen, sondern Gründe dafür ausfindig zu machen, warum es auf diese Frage keine Antwort gibt. Es muss also zwischen Gott und der Theologie unterschieden werden. Allerdings macht die eben kurz skizzierte Situation die Theologie, wenn sie bei ihrer Sache bleibt, bescheiden.

Blicken wir jetzt von der Theologie noch einmal hin auf den Gott, von dem sie redet, so können wir noch einiges Klärende zur Bezeichnung Gottes als eines kommunikativen Wesens beitragen. Für Glaubende gilt, dass Gott selbst ein Beziehungswesen ist. Diese Vorstellung ist keine Erfindung von Menschen, sondern wird erst ermöglicht durch die Selbstoffenbarung Gottes; das heißt dadurch, dass Gott eine Beziehung zur Welt als Schöpfung und zu den Menschen und seinen Geschöpfen als Sündern und Erlösten und der Vollendung Entgegengehenden eingeht; nur deshalb ist von ihm als von einem Gott in Beziehung zu reden. Über Gott an und für sich können wir auch in der christlichen Theologie keine Aussagen machen. Gott an und für sich begegnet uns immer nur als der Gott/ wr uns. Daraus lässt sich umgekehrt nicht folgern, dass es Gott an und 
für sich nicht gäbe, weil er nur als Gott für uns erscheint. Wohl aber ist dadurch eine Grenze unserer theologischen Reflexions- und Sprachmöglichkeit gezogen. So können wir im Blick auf das Konzept der Kommunikativen Theologie sagen: Nur weil Gott eine Beziehung mit uns eingeht, weil er mit uns Gemeinschaft haben will und mit uns Kommunikation aufnimmt, deshalb können wir überhaupt von Gott, zu Gott und über Gott reden. Insofern könnten wir auch unsere Bezeichnung noch präzisieren, indem wir von Gott als dem kommunikativen Wesen sprechen. Gott ist es, der zu allererst die Kommunikation, die Gemeinschaft, die uns am Leben erhält, ermöglicht. Dies ist unser Glaube an Gott den Schöpfer von Himmel und der Erde, der sichtbaren und der unsichtbaren Welt. Nicht erst das, was wir die Offenbarung Gottes in der Geschichte nennen, sondern schon seine Offenbarung als Schöpfergott erweist ihn als kommunikatives Wesen, als das kommunikative Wesen.

Unabhängig von der Selbstoffenbarung Gottes können wir nämlich über ihn keine verlässliche Aussage machen. Deshalb ist das Missverständnis abzuwehren, wir könnten hier quasi eine Theologie von oben her betreiben, d. h. uns auf die Ebene Gottes begeben und zunächst einmal einen Gottesbegriff an und für sich entwickeln, um ihn dann nachträglich mit der Geschichte in Verbindung zu bringen. Wenn wir also, bevor wir auf Offenbarung und Geschichte eingehen, bereits über Gott als das kommunikative Wesen reden, so haben wir den Ausgangs- und Ermöglichungspunkt unseres Redens nie aus dem Blick verloren.

Unter dieser Voraussetzung - dass wir von, zu und über Gott nur reden können, weil Gott zu uns geredet hat und redet - können wir noch einen weiteren Schritt tun, der für die christliche Theologie ebenso zentral wie in ihr selbst umstritten ist. Dass Gott im Blick auf Schöpfung und Menschheit als kommunikatives Wesen, als ein Gott in Beziehung verstanden werden darf, ist klar geworden. Christliche Gottesverkündigung spricht darüber hinaus auch von Gott als einem in sich beziehungsreichen Wesens. Dies ist der Kerngehalt des christlichen Glaubens an den Dreieinigen Gott, der als solcher höchste Ansprüche an eine theologische Explikation stellt. Wir haben in unseren Kursen zur Kommunikativen Theologie diesen Kerngehalt auf den Punkt zu bringen versucht, in dem wir die einschlägigen Kurse zur Kommunikation des Dreieinigen Gottes überschrieben haben. Im Ursprung ist Beziehung. Dieses Motto gilt im Blick auf Schöpfung und Menschheit in jedem Fall, wie bislang ausgeführt wurde. Gilt es auch im Blick auf Gott? Müssen wir bzw. können wir bzw. dürfen wir sagen, dass Gott, der Ursprung von allem, in sich selbst ursprünglich ein Beziehungswesen, ein kommunikatives Wesen ist? Abstrakte Spekulationen über den Gottesbegriff helfen hier nicht weiter. Im Blick auf das Gespräch mit den monotheistischen Religionen des Judentums und des Islam ist zu sagen, dass Gott im Blick auf die Schöp- 
fung und die Menschheit ein kommunikatives Wesen, ein Beziehungswesen sein kann, auch wenn er in sich streng monotheistisch gedacht wird, wenn also nicht von Beziehungen in Gott geredet wird. Wie kommt aber nun die christliche Theologie dazu, von Beziehungen in Gott zu reden?

\section{Lebendige Überlieferung als Kommunikationsprozess}

Wir hatten bereits bei der Kennzeichnung des Offenbarungsverständnisses des Ersten Vatikanischen Konzils darauf hingewiesen, dass Offenbarung, wenn sie vor allem als Mitteilung von zu glaubenden Lehrsätzen verstanden wird, vornehmlich in der geschriebenen und überlieferten Offenbarung zu finden ist. Selbstverständlich gilt die Heilige Schrift als die Urkunde des Glaubens und als die von keiner anderen Instanz mehr zu normierenden Norm. Dies gilt auch in der erneuerten Sicht des Offenbarungs- und Glaubensverständnisses, welche das Zweite Vatikanische Konzil sich zu eigen gemacht hat. Freilich wird die Heilige Schrift nicht in erster Linie als Sammlung von Lehrsätzen verstanden, aus der man schöpfen könne, um Lehren der Kirche als schriftgemäß zu erweisen. Vor allem ist die Überlieferung, die Tradition der Kirche, nicht eine zweite weitere materiale Quelle, aus der wiederum Autoritätsbeweise für die Richtigkeit der kirchlichen Lehre gewonnen werden könnten. All dies gilt auch, wird aber im Sinne des Zweiten Vatikanischen Konzils relativiert, in dem es eingeordnet wird in den größeren Zusammenhang einer kommunikatorisch-partizipatorisch verstandenen Offenbarung. In der Auseinandersetzung zu Zeiten des Ersten Vatikanischen Konzils war es wichtig geworden, wer die entscheidende (unfehlbare) Autorität in der Kirche darstellt. Im Zusammenhang mit dem kommunikatorisch-partizipatorischen Offenbarungsverständnis des Zeiten Vatikanischen Konzils hat die Theologie die Vielfalt der Subjekte wieder entdeckt. In der Fachsprache spricht man wieder von mehreren loci theologici. Dabei wird zurückgegriffen auf den neuzeitlichen Theologen Melchior Cano, der in der Auseinandersetzung der Reformationszeit auf die verschiedenen Bezeugungsinstanzen der Offenbarung und des Glaubens hingewiesen hat. Neben der ungeschriebenen Überlieferung Christi und der Apostel sind dies z. B. die Gesamtkirche (ecciesia catholica), die altkirchlichen Konzilien, die römische Kirche, Kirchenväter, die Theologen überhaupt, das Lehramt. Neben den eigentlichen loci theologici gibt es auch solche, die nicht genuin theologisch sind, jedoch in der Theologie „Bürgerrecht genießen": die natürliche Vernunft, die Geschichte und die Philosophien und die menschliche Geschichte ${ }^{13}$. Wir werden im Kapitel über das ge-

13 Vgl. Seckler, M., Loci theologici, in: LThK, Bd. 6 (1997) 1014-1016. 
schenkte Wir der Kirche noch auf die Unterscheidung und das In Beziehung Stehen der verschiedenen Subjekte der Glaubenskommunikation zu sprechen kommen.

In unserem Zusammenhang ist wichtig festzuhalten, dass Überlieferung (Tradition) im Sinne des Zweiten Vatikanischen Konzils als ein lebendiger Prozess verstanden wird. Wir sprechen nicht mehr von zwei materialen Quellen der Offenbarung, der Bibel und der Tradition, sondern von der Überlieferung des in der Urkunde der Heiligen Schrift bezeugten apostolischen Glaubens als Antwort auf die Selbstmitteilung Gottes in Jesus Christus in seinem Geist. Das Wort der Schrift legt sich nicht selbst aus, sondern muss verkündet und im Hören und Lesen und im Tun lebendig werden. Dies ist der Überlieferungsprozess der Glaubensgemeinschaft Kirche. Weil es in der Tradition um die Weitergabe des Wortes des Lebens geht (vgl. den Anfang des ersten Johannesbriefs), deshalb muss auch der Traditionsprozess ein kommunikatorischer-partizipato-rischer Prozess sein. Hier hat das Zweite Vatikanische Konzil in der neuen Perspektive erste Schritte unternommen. Diese bleiben freilich auch in ökumenischer Hinsicht noch im Anfangsstadium dessen, was ein kommunikatorisch-partizipatorisches Überlieferungsverständnis bedeuten könnte. Aber das Konzil hat die Tür aufgetan, hier sind jetzt entschieden Schritte weiterzugehen.

\section{Das Spezifikum Kommunikativer Theologie}

Glaubenspraxis als Ausgangspunkt, als bleibender Bezugs- und Zielpunkt theologischer Arbeit - ja! Aber warum sollen wir diese Glaubenspraxis kommunikative Praxis nennen?

Der unterschiedliche Gebrauch des Wortes Praxis führt gerade auch in innerkirchlichen und innertheologischen Diskussionen immer wieder zu Missverständnissen. Die Bandbreite möglicher Einstellungen reicht von der Gruppe derer, die alle Theorie grundsätzlich für praxisfeindlich halten, bis zu denjenigen, welche die praktische Verzweckung und damit den Abbau der Wissenschaftlichkeit der theologischen Theorie fürchten. Wir gehen hier von einem Praxisbegriffaus, der die relevanten Lebensäußerungen der Menschen, besonders der Menschen in der Kirche, der Theologinnen und Theologen umfasst. Was gemeint ist, lässt sich im Blick auf einer nach dem Zweiten Vatikanischen Konzil immer wieder geführten Debatte verdeutlichen. Haben die Laien Anteil bloß am Weltdienst der Kirche oder auch an ihrem Heilsdienst, so lautete die Alternativfrage. Sie ist eine typische Frage der nachkonziliaren Diskussion, und als solche ist sie ebenso typisch für eine von vornherein schief gestellte Alternative. Im Sinne des Zweiten Vatikanischen Konzils müssen wir ge- 
rade sagen: Alles, was Christenmenschen aus dem Glauben heraus tun, ist Heilsdienst. Auch der Weltdienst, verstanden als Dienst an der Welt und den Menschen, ist, wenn er aus Glauben heraus geschieht. Heilsdienst. So könnten wir im Blick auf unsere Arbeit festhalten, dass wir unter Praxis alles das verstehen, was Christenmenschen, ja Menschen überhaupt, glaubensrelevant tun.

Theologie wird vielfach als "Glaubenswissenschaft” bezeichnet. Dies ist missverständlich, weil sie - oft nur im eingeschränkten Sinne - als eine bloß kognitive Erkenntnisleistung verstanden wird. In diesem Fall meint man mit "Glaube" ausschließlich das Fürwahrhalten von Glaubenssätzen. Glaube im umfassenden Sinn ist das, was eben als Praxis erläutert wurde: das Leben/ Handeln von Christen-innen als glaubenden Menschen. Insofern könnte die Theologie als Glaubenspraxiswissenschaft bezeichnet werden. Um einerseits die Missverständlichkeit des Wortes Glaubenswissenschaft und andererseits die Umständlichkeit der Formulierung Glaubenspraxiswissenschaft zu vermeiden, empfiehlt sich die Rede von der Kommunikativen Theologie. Spätestens jetzt ist ein klärendes Wort zu dem notwendig, was mit "kommunikativ" in diesem Zusammenhang gemeint ist.

In einer ersten Klärung empfiehlt es sich, von einer Unterscheidung auszugehen, die der soeben mit dem Friedenspreis des deutschen Buchhandels ausgezeichnete Philosoph Jürgen Habermas vorgenommen hat. Habermas, der zunehmend die Bedeutung der (Ausdruckweisen, nicht des Wahrheitsgehaltes von) Religion für die menschliche Praxis entdeckt, unterscheidet zwischen einer instrumentellen und einer strategischen Praxis auf der einen und einer kommunikativen Praxis auf der anderen Seite $^{14}$. Beide Seiten unterscheiden sich durch die Art der jeweils beanspruchten Rationalität und ihr Ziel: Im ersten Fall geht es um instrumentelle Verfügung, im zweiten um kommunikative Verständigung. Die Formen kommunikativer Praxis sind durchaus vielfältig. Entscheidendes Kriterium für eine christliche Communio ist ja die Ausrichtung auf verbale wie nonverbale Kommunikation, wie sie sich in einer konkreten Communio zeigt. So ist keine mögliche Handlung von vornherein als irrelevant ausgeschlossen, vielmehr ist das Kriterium für die Qualität einer Handlung der Bezug zur Kommunikation innerhalb dieser Gemeinschaft.

Nun ist freilich noch einmal von dieser Seite her klar geworden, dass Kommunikation nicht auf verbale Äußerungen reduziert werden darf. Alles, was der Stiftung, der Aufrechterhaltung und der Vollendung der Glaubensgemeinschaft dient, ist als kommunikative Handlung zu bezeichnen. (Diese weite Formulierung deutet schon an, dass es im Blick auf die Communio der Glaubenden auch kommunikative Handlungen gibt, deren Subjekte nicht Menschen sein müssen oder können.) 
Eine Rangordnung kommunikativer Akte in der Glaubensgemeinschaft kann nicht von den zu unterscheidenden menschlichen Subjekten ausgehen (dass kommunikative Handlungen, die Gott zum Autor haben, davon deutlich zu unterscheiden sind, gehört zum Selbstverständnis dieser Glaubenskommunikation); nicht wer die jeweilige Handlung ausführt, sondern wozu sie dient, entscheidet über ihren Rang. In diesem Sinne kann man unter der kommunikativen Praxis kommunikative Handlungen besonderer Art ausmachen. In einer gewissen Anknüpfung an den eben referierten Sprachgebrauch hat Peter Hünermann von kommunikativen Handlungen gesprochen, die jede gesellschaftliche Gruppe zur Aufrechterhaltung ihrer selbst benötigt. Er formuliert:

„Kommunikative Handlungen unterscheiden sich dadurch von anderen Tätigkeiten, dass sie auf andere Menschen ausgerichtet sind und sie mit ihrer geschichtlichen Freiheit in die Handlung einbeziehen. Unter den vielfältigen kommunikativen Handlungen gibt es einige, die für eine bestimmte Gruppe konstitutiv sind. In und durch ihren Vollzug entsteht und erhält sich die Gruppe. Ohne diese kommunikativen Handlungen gäbe es auch die Gruppe nicht. Bei diesen konstitutiven Handlungen handelt es sich um Figuren gemeinsamen Lebens. Nur mittels dieser Figuren erbildet sich ein solches Leben. Nur im immer erneuten Vollzug dieser Figur des Lebens bleibt es bestehen ${ }^{\prime 15}$.

Es erstaunt nicht, dass P. Hünermann diesen anthropologischen Ansatz zur Erläuterung dessen wählt, was wir unter einem Sakrament verstehen. Sakramente sind ausgezeichnete kommunikative Handlungen, die konstitutiv für die Glaubensgemeinschaft sind. Das heißt: Eine Glaubensgemeinschaft, die sich nicht mehr zu Gebet und sakramentalen Feier trifft, höhlt sich von innen her aus. Gerade die Sakramente machen aber noch einmal deutlich, dass konstitutive kommunikative Handlungen Handlungen sind, die in einem ganzheitlichen und umfassenden Sinn der Auferbauung der Glaubensgemeinschaft dienen. Nicht weniger wahr ist die schlichte Formulierung, dass kommunikative Handlungen solche sind, in denen Menschen sich wechselseitig zum Menschsein verhelfen.

Wir nennen nun die spezifische Art unseres Theologietreibens "Kommunikative Theologie". Weil in jeder Theologie kommuniziert wird, erfasst eine „Kommunikative Theologie” alles, was Theologie überhaupt ausmachen kann. Aber nicht jede Theologie ist ausdrücklich auf die kommunikative Glaubenspraxis des Gottesvolkes ausgerichtet, nicht jede Theologie macht diesen Kommunikationsprozess explizit zum Bezug-

14 Vgl. das einschlägige Grundlagenwerk: Habermas, Jürgen, Theorie des kommunikativen Handelns, 2 Bde., Frankfurt a. M. 1999.

15 Hünermann, p., Sakrament - Figur des Lebens, in: Schaeffler R., Hünermann P., Ankunft Gottes und Handeln des Menschen (QD 77), Freiburg i. Br. 1977, 55. 
spunkt ihrer Reflexion. Wenn das Konzept der Kommunikativen Theologie wohl alle Bereiche der Theologie umfasst, so ersetzt sie aber dennoch nicht andere Konzepte wie z. B. die Theologie der Befreiung oder die feministische Theologie. Der Grund dafür liegt in der Perspektive, die andere Theologien zusätzlich ins Blickfeld bringen.

So wird in der Theologie der Befreiung eine bestimmte Perspektive der Arbeit von Theologie eröffnet, die eine Kommunikative Theologie gerade nicht ausschließt, wenn sie das, was in der Theologie zur Sprache kommt, in der Perspektive des Gottes, der befreit, betrachtet. Insofern stellt die Befreiungstheologie eine Spezifikation, eine Konkretisierung dessen dar, was Thomas von Aquin, den auch noch das jüngste Konzil als Lehrmeister für die Theologiestudierenden empfahl ${ }^{16}$, beschrieben hat: In der Theologie geht es darum, alles sub ratione Dei, also im Blickwinkel Gottes zu betrachten. Theologie als Logos von Theos, als Rede von Gott, spricht also nicht über Gott, sondern im wahrsten Sinne des Wortes über Gott und die Welt, über die Welt aber in der Perspektive Gottes. Befreiungstheologie tut dies in der Perspektive des befreienden Gottes, feministische Theologie in der Perspektive vor allem der Gotteserfahrung von Frauen. Es sind jeweils geschichtlich bedingte Situationen, die dazu führen, die Arbeit der Theologie in einer besonderen Perspektive zu orientieren. Karl Rahners transzendentale Theologie ${ }^{17}$ war der Versuch, die steril gewordene neuscholastische Schultheologie dadurch zu überwinden, indem er nach den Bedingungen der Möglichkeit für den Glauben der Menschen fragte. Transzendental ist der Fachausdruck für diese Reflexion auf die Bedingungen der Möglichkeiten: Was kann den Menschen in seiner Selbsterfahrung und seinem Selbstvollzug dazu bewegen, dem Glauben an die Menschwerdung Gottes Glauben zu schenken?

Eine andere bedeutende theologische Figur des 20. Jahrhunderts war die dialektische Theologie, die in erster Linie von Karl Barth begründet wurde $^{18}$. Karl Barth legte nach dem Zusammenbruch des Kulturprotestantismus und der liberalen Theologie, also dem Kollaps bestimmter Ausprägungen der abendländischen Kultur nach dem Ersten Weltkrieg, den Schwerpunkt auf die radikale Unterschiedenheit von Gott und Mensch, das radikale Ausgeliefert sein des Menschen an Gott. Die Spannung zwischen dem Ja Gottes und dem Nein des Menschen bezeichnete er mit „Dialektik”. In den Religionen sah K. Barth, zumindest in der frühen Phase der dialektischen Theologie, das vergebliche, ja sogar sündige

16 Vgl. das Dekret über die Ausbildung der Priester sowie „Optatam totius” (OT) Nr. 16.

17 Vgl. Propper T., Striet M., Transzendentaletheologie, in: LThK3, Bd.10 (2001) 188-190.

18 Vgl. Barth K., Der Römerbrief, 2. Fassung, München 1922 (Zürich „1999); Molt-Mann J. (hrsg.), Anfänge der Dialektischen Theologie, Bd. 1, München 1995. 
Bemühen des Menschen, an Gott heranzureichen. Zu jeder menschlichen Religion sagt die Offenbarung Gottes ein radikales Nein, weshalb Barth sogar lange meinte formulieren zu müssen, dass es keinen Anknüpfungspunkt für die Offenbarung Gottes im Menschen gäbe. Später musste er dies revidieren, denn die Offenbarung Gottes braucht selbstverständlich einen Adressaten, dem Gott sich offenbart. Also zumindest als Empfänger der Botschaft muss der Mensch konstituiert sein. Auch hier sehen wir - und deshalb dieser kleine Ausflug in die Theologiegeschichte -, wie unentbehrlich das Konzept der Kommunikation als Beziehung von Subjekten ist, um Theologie und das, was in Theologie geschieht, zu verstehen.

Kommunikative Theologie ist also diejenige Theologie, welche die kommunikative Praxis der Communio der Glaubenden reflektiert. Eine solche Theologie muss selbst als ein kommunikatives Geschehen begriffen werden. Das schließt nicht aus, dass nach wie vor auch schweißtriefende Arbeit am Schreibtisch, in der Bibliothek oder in Archiven verlangt ist. Aber im Blick auf das Ganze der Theologie fügt sich alle Einzelarbeit zusammen in den kommunikativen Akten der Begegnung, der Diskussion, der Disputation usw.

\section{Początki teologii komunikacji}

\section{STRESZCZENIE}

Pojęcie komunikacja, od łac. „communicatio” - porozumiewanie się, dzielenie się z kimś czymś, informowanie, etc. jest z natury międzyludzkim, osobowym spotkaniem i porozumieniem za pomocą werbalnych czy nie werbalnych znaków. Człowiek jako „istota dialogowa” skazany jest na komunikowanie się, aby móc żyć i w ogóle przeżyć tak psychicznie jak i fizycznie. Dzięki komunikacji może człowiek być i stawać się we wspólnocie. Komunikacja ma zatem tworzącą i podtrzymującą moc. Komunikacja wiary sprawia „otwarcie się nieba" i przystęp do Boga. Filozoficzno-socjologiczne pojecie komunikatywnego działania jest rozmaicie interpretowane. Jest jednak otwarte na podstawowe dialogowe rozumienie człowieka jako wyraz jego kreatywności, Bożego podobieństwa i wolności. Odpowiednio do tego człowiek jest zawsze i ostatecznie przez Boga „zagadywaną istotą", w wolności odpowiadającą, ale również mogącą nie reagować na Boże inspiracje. Rozumienie komunikacji radykalizuje się w obliczu wspólnoty komunikacji, która w chrześcijańskiej nadziei ogarnia także zmarłych. Wprawdzie żyjący sami nic dla umarłych uczynić nie mogą, ale poruszają ratującą ich rzeczywistość Boga.

Współczesność komunikacji nie wyczerpuje się już dawno tylko w „werbalizmie słowa czy mowie ciała”. Możliwości i rozmiar komunikowania są w przełomowym momencie dziejów i rozwijają się w niespotykanym tempie. Fu- 
turolodzy mówią o „placeless society” - społeczeństwie bez miejsca na ziemi. Czas i przestrzeń w nowoczesnej komunikacji nie będą odgrywały już istotnej roli. Sieć informatyczna urasta do „pępka świata”. Jeszcze w XXI wieku państwa narodowe, firmy, związki zawodowe i polityczne w dzisiejszej formie przestaną istnieć podobnie jak szkoły, religie czy rodziny. Do największych przegranych tego procesu należą już dzisiaj ci, którzy niewystarczająco wcześnie włączyli się w globalną sieć komunikacji lub są informatycznymi analfabetami. Rozwój globalnego i wirtualnego społeczeństwa komunikacji spowoduje zmiany w tożsamości człowieka.

Paul Watzlawick, badacz procesów komunikacji wypowiada pewnik „człowiek, niezależnie od tego jak, ale "nie może nie komunikować". Odpowiada to naszemu doświadczeniu od początku życia. W ludzkiej komunikacji, jeśli chce być godna miana człowieczej, to ludzie winni odkrywać i spotykać ludzi. Człowiecza komunikacja jest relacją , ja - ty" lub ,ja - my". Martin Buber powiada: „pedagogicznie owocny nie jest pedagogiczny zamyst, ale pedagogiczne spotkanie". Człowiek nie może stać się nigdy w człowieczej komunikacji obiektem „czymś”. Bóg jest Subiektem, wyjściowym i trwałym punktem odniesienia każdej teologicznej refleksji. Teologia komunikacji stawiająca pierwsze kroki pragnie odnieść się do rozumienia komunikacji jako integralnej mowy o Bogu w komunikacji z Bogiem, która tworzy z Nim więź. Dla ludzi wiary Bóg sam jest Osobą więzi relacji osobowych. Osiągnięcia techniczne komunikacji i komunikowania stają się wyzwaniem dla filozofów i teologów. Powyższy artykuł jest skromną tego próbą. 